\title{
Concept of Ideal Closure Line for Clipping of Middle Cerebral Artery Aneurysms
}

\author{
-Technical Note-
}

\author{
Tatsuya ISHIKAWA, Naoki NAKAYAMA*, Junta MOROI, Norikata KOBAYASHI, \\ Hideya KAWAI, Tastushi MUTO, and Nobuyuki YASUI
}

\begin{abstract}
Department of Surgical Neurology, Research Institute for Brain and Blood Vessels-Akita, Akita;
*Department of Neurosurgery, Hokkaido University Graduate School of Medicine, Sapporo, Hokkaido
\end{abstract}

\begin{abstract}
The concept of optimum closure line was applied to a series of 51 consecutive middle cerebral artery aneurysms (14 ruptured, 37 unruptured) in 41 patients, 16 men and 25 women aged 29-79 years (mean 59.1 years). Visual inspection through the operating microscope revealed 3 types of aneurysm based on the origin of the aneurysm: bifurcation type $(n=39)$, trunk type $(n=9)$, and combined type $(n=3)$. Clipping along the optimum closure line should restore the vascular structure to the original configuration. Combination clip techniques were useful to form a curved closure line. This technique requires adequate operative fields with dissection of the aneurysm and related arteries from the neighboring structures as far as possible. The closure line concept is helpful to decide how to apply clips for particular aneurysms to avoid risks of ischemic complication and future recurrence. Combination clip techniques are often necessary to match a curved closure line.
\end{abstract}

Key words: cerebral aneurysm, clipping surgery, neck remnant, regrowth

\section{Introduction}

Clipping surgery has been a standard and reliable technique to exclude cerebral aneurysms from the circulation and prevent bleeding since the 1950s. ${ }^{2,14,18)}$ Clipping of aneurysms is aimed at obliterating the three-dimensional (3D) orifice of the aneurysm and recreating a curved or straight line with the blades of the aneurysm clip. However, the clip shape does not always match the neck shape despite the variety of commercially available aneurysm clips. ${ }^{8111}$ Both de novo aneurysm and regrowth of a previously clipped aneurysm are potential origins of subarachnoid hemorrhage. . $^{1,4,6,7,9,13,15-17)}$ Postoperative 3D computed tomography angiography and conventional angiography indicate that $5.9-42 \%$ of residual aneurysm persists after clipping. ${ }^{6,13)}$ A small remnant is thought to pose little risk, ${ }^{13)}$ but residual neck can dilate and form a dangerous aneurysm after a long period, even if the remnant is only $1-2 \mathrm{~mm}$ in size. ${ }^{9)}$ Late follow-up angiography suggests that $25 \%$ of aneurysms with dog-ear remnant and $75 \%$ with broad-based remnant show regrowth. ${ }^{4}$ Endovascular treatment after clipping surgery may resolve the remnant aneurysm, ${ }^{12}$ but complete obliteration at the primary surgery is preferable. However, occlusion of the parent arteries by inappropriate clip placement must be avoided. ${ }^{18)}$

The procedures of clipping have been extensively described, ${ }^{2,3,14,18)}$ but practical application depends on the learning of surgical skills by observing operations. In particular, the performance of optimum clipping to avoid surgical complications and future recurrence has not been formally described. We recently proposed the concept of closure line based on the shape of aneurysm origin as a guide to optimum clipping. ${ }^{5}$

The present study analyzed a series of middle cerebral artery (MCA) aneurysms treated by clipping according to the closure line based on the shape of the aneurysm orifice to evaluate the effectiveness of the concept. 


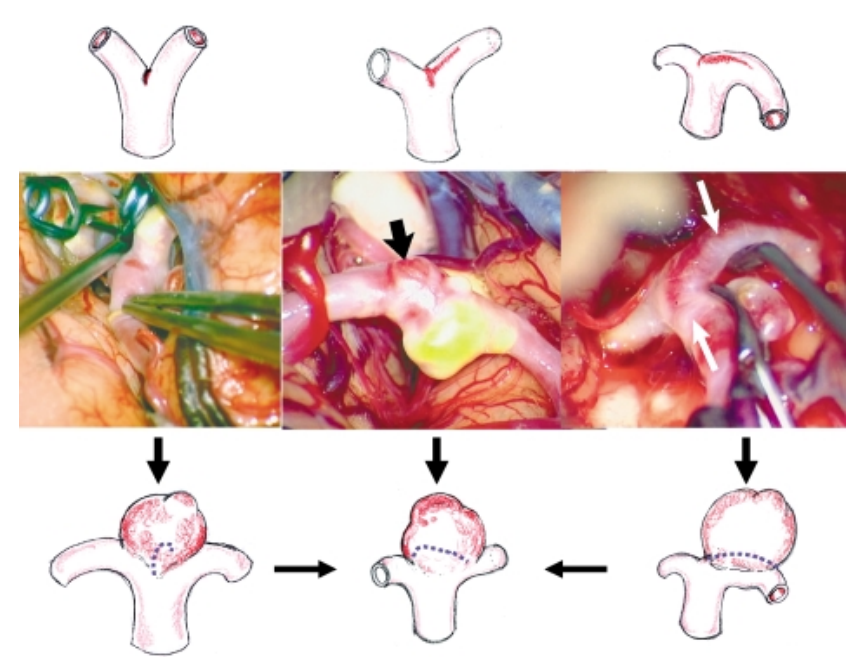

Fig. 1 Diagrams and photographs illustrating the different types of aneurysm configuration. Left column: The bifurcation type aneurysm seems to originate from a curved linear cleft at the bifurcation (upper) shown as an abnormal thin wall running perpendicular to the longitudinal axis of the efferent artery in the operative photograph (middle). Right column: The trunk type aneurysm has an orifice on the efferent artery and originates from loss of the tunica elastica on one of the efferent arteries (upper). The operative photograph shows a clipped trunk type aneurysm, and abnormal thin wall on the efferent arteries on both sides (middle, white arrows). Center column: The combined type aneurysm has components of both types (upper). Growth of the aneurysm may destroy the tunica elastica of the continuous vessels and the aneurysm eventually enlarges. The operative photograph shows a bifurcation type aneurysm with sclerotic wall associated with change in the vascular wall of one of the efferent arteries (middle, thick arrow). Lower row: Blue dotted lines show the ideal closure lines, appearing perpendicular, parallel, and oblique to the longitudinal axis of the efferent artery, respectively.

\section{Surgical Technique}

A series of 51 consecutive cases of MCA aneurysms of less than $15 \mathrm{~mm}$ in diameter (14 ruptured, $37 \mathrm{un}$ ruptured) was analyzed in 41 patients, 16 men and 25 women aged 29-79 years (mean 59.1 years) treated in the Research Institute for Brain and Blood Vessels-Akita between November 2006 and December 2007. Visual inspection through the operating microscope revealed 3 types of aneurysm according
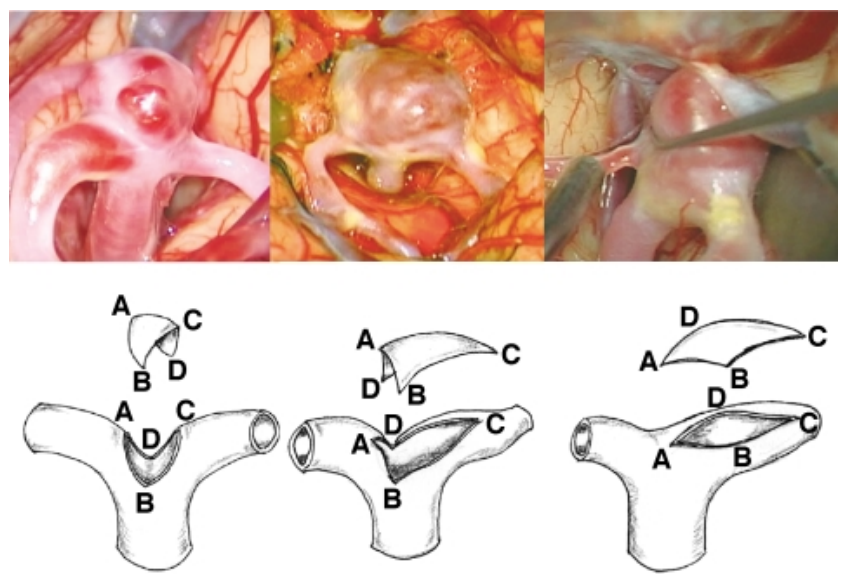

Fig. 2 Representative photographs (upper row) and illustrations (lower row) of the 3 different types of aneurysm configuration. Left column: The bifurcation type aneurysm has an orifice with a deeply curved surface and 4 vertices (A-D). The aneurysm is considered to have originated from a cleft corresponding to the curved line $B D$, and to have opened the orifice along lines BAD and BCD. Right column: The trunk type aneurysm has an orifice with a relatively flat surface and 4 vertices (A-D). The aneurysm is considered to have originated from the cleft corresponding to the line AC, parallel to the longitudinal axis of the efferent artery, and to have developed along lines ADC and ABC separating from each other. Middle column: The combined type aneurysm has an orifice with a curved surface and 4 vertices (A-D). The aneurysm is considered to have originated from a $T$ shape cleft (lines BAD and AC) and to have developed along lines $\mathrm{AB}, \mathrm{BC}, \mathrm{CD}$, and DA.

to the origins. ${ }^{5)}$ The most common type was the bifurcation type $(\mathrm{n}=39)$ of $1-11 \mathrm{~mm}$ diameter (mean $6.4 \mathrm{~mm}$ ), which apparently originated from a curved linear cleft originating at the bifurcation of the arteries (Fig. 1 left column), with an orifice forming a deeply curved rhombus (Fig. 2 left column). The second type was the trunk type $(\mathrm{n}=9)$ of $1-11 \mathrm{~mm}$ diameter (mean $5.0 \mathrm{~mm}$ ), which appeared to originate from loss of the tunica elastica on one of the efferent arteries (Fig. 1 right column), with an orifice forming a flat rhombus on the parent artery (Fig. 2 right column). The third type was a combined type $(\mathrm{n}=3)$ of 6-12 mm diameter (mean $8.7 \mathrm{~mm}$ ), in which growth of the aneurysm destroyed the tunica elastica of the continuous vessels with subsequent enlargement (Fig. 1 middle column), with an orifice forming a curved trapezoid (Fig. 2 middle column). 


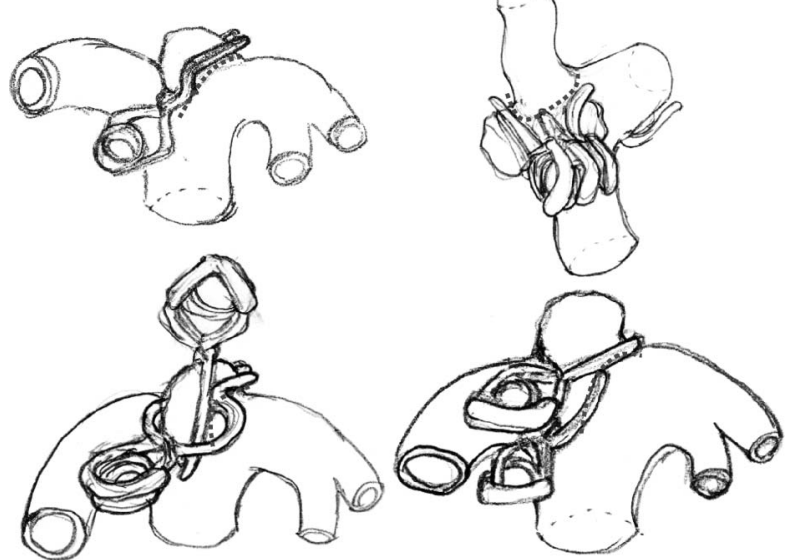

Fig. 3 Illustrations showing clipping of the entire neck of the bifurcation type aneurysm, in which the orifice has a deeply curved surface. Use of a crank-shaped clip (shank clipping, upper left), back-to-back placement (back-to-back clipping, upper right), a fenestrated clip across the primary clip (cross clipping, lower left), or a second clip placed under the primary clip (under clipping, lower right). Blue dotted lines represent the ideal closure lines.

The closure line is defined as the line on the aneurysm emerging at clipping. ${ }^{5)}$ If the closure line restores the original vascular configuration present before the aneurysm developed, the maximum of the pathological wall of the aneurysm is included within the clips. Such a closure line is termed the ideal closure line. The ideal closure line is perpendicular to the efferent artery in the bifurcation type aneurysm and parallel in the trunk type aneurysm. The ideal closure line is theoretically a T-shape for combined type aneurysms, and is impossible to achieve by clipping. However, the oblique clip offers practical solutions for the combined type aneurysm.

Clip application parallel to the efferent artery necessarily causes broad-based residual neck in the case of the bifurcation type aneurysm. Clip application perpendicular to the efferent artery often results in dog-ear residual neck. Therefore, we must consider that the actual aneurysm orifices have a curved surface, particularly with bifurcation type and combined type aneurysms. The ideal closure line is thus a curved line for these types of aneurysm. Tandem clipping has been used in combination with the primary clip to eliminate such dog-ear remnants. Different methods are available, under, cross, and back-to-back clipping, to place the second or third clips, depending on the relationship with the primary clip (Figs. 3 and 4). Placement of additional clips
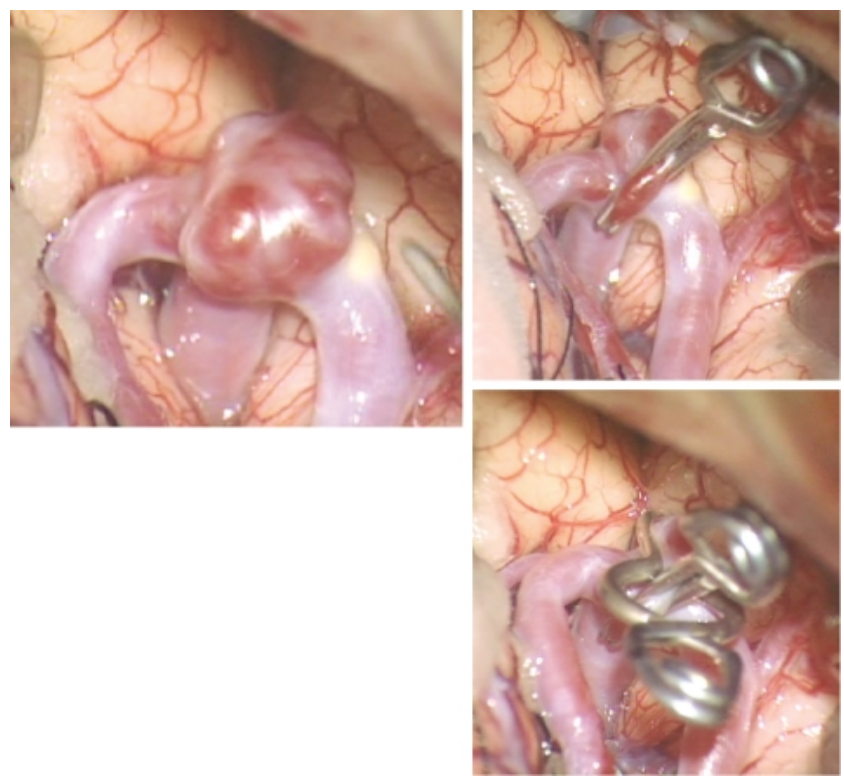

Fig. 4 Photographs showing clipping for a bifurcation type aneurysm (unruptured). The aneurysm orifice has an extremely curved surface (left). The primary clip was applied to cover the thinner part of aneurysm wall with a J-shaped clip (right upper), and then the remnant was closed with a fenestrated clip applied across the primary clip (right lower).

on the primary clip is useful to obliterate the aneurysm from the circulation completely. Shank clipping using the shank of a bayonet-type clip is also effective to close the entire neck of such aneurysms, ${ }^{8,11)}$ but the shank does not always closely match the shape of the neck.

Selection of combination under or cross clipping should be based on the shape and location of the remnant after primary clip placement. Indeed, we are forced to place second and third clips in the same direction as the primary clip. Therefore, we should carefully consider the final anatomical configuration before placing the primary clip on the aneurysm. Clip application along the ideal closure line enables elimination of the entire neck with minimal remnant. Full mobilization of the aneurysm and parent arteries is occasionally necessary to match the direction of clip application with the ideal closure line. ${ }^{5)}$

Many MCA aneurysms occur over the insular cortex, so complete dissection of the aneurysm from the surrounding neuronal structures and neighboring vessels can provide sufficient space to mobilize the aneurysm to clip along the ideal closure line. However, if the aneurysm originates from a short $M_{1}$ and is located around the limen insulae, the possible 
directions for insertion of the clip applicator are very limited, and clipping away from the ideal closure line must be performed even for bifurcation type aneurysms. In such cases, occlusion of the parent arteries must be avoided by relatively loose clip application to the neck. Moreover, additional efforts to remove the residual neck are necessary, such as change of the application angle by rotation of the aneurysm with the left hand, temporary clipping, and coating of the remnant.

In our series, clips for the 39 bifurcation type aneurysms were applied perpendicular to the efferent arteries along the ideal closure lines in 26 aneurysms, and parallel away from the ideal closure lines in 13. Clips for the 9 trunk type aneurysms were placed parallel to the efferent arteries along the ideal closure lines in 5 aneurysms, perpendicular to the efferent artery away from the ideal closure lines in 2, and obliquely in 2. Clips for the 3 combined type aneurysms were all placed obliquely. Substantial parts of the aneurysms with arteriosclerotic changes around the aneurysm and neighboring arteries were not intentionally included within the clips to avoid ischemic complications.

\section{Discussion}

MCA aneurysms often have particularly complex shapes of the aneurysm neck, so clipping surgery is preferred over endovascular treatment. ${ }^{3)}$ Several techniques have been recommended to achieve optimum clip placement for such complex aneurysms with a broad, irregular base and arteriosclerotic or thrombotic sac, such as step-wise elimination, bipolar coagulation, and temporary clipping. ${ }^{18)}$

Coagulation of the aneurysm dome is particularly useful for shaping the neck of the aneurysm before clipping. ${ }^{18)}$ Bipolar coagulation is also effective for the treatment of microaneurysms not amenable to clipping. ${ }^{10)}$ However, this procedure carries some risk of aneurysm rupture as well as irreversible injury to the parent and perforating arteries. ${ }^{10,18)}$ In addition, there is no histological conformation allowing bipolar coagulation to reinforce the aneurysm wall. ${ }^{10)}$ Many MCA aneurysms are treated by resident neurosurgeons, because the approach and handling of the aneurysm are relatively simple. Even if supervised by senior neurosurgeons, we should avoid maneuvers that risk mortality and morbidity.

The present study applied our concept of aneurysm type and ideal closure line to eliminate the aneurysm as far as possible. ${ }^{5}$ A combination clip technique based on the concept of the closure line may offer a safe and rational way to achieve com- plete clipping. The present study found that bifurcation type aneurysms had an orifice with a deeply curved surface, and so required a deeply curved closure line, which required the application of additional under or cross clips. Additional clip placement for a dog-ear remnant might cause avulsion of the thinner wall. ${ }^{10)}$ Therefore, we applied the primary clip to include the thinner part of the aneurysm wall in the primary clip. The dog-ear remnant produced by the primary clip may thus have a relatively thicker wall, and can be safely occluded with a second clip (Fig. 4).

The present study included only MCA aneurysms of less than $15 \mathrm{~mm}$ in diameter, so this experience cannot be applied to large aneurysms, in which the original shape of vascular structure has been lost. Arteriosclerotic changes around aneurysm and its neighboring arteries also prevented application of clips along the ideal closure line. Safe aneurysm closure without ischemic complication is preferable, even if a substantial remnant of the aneurysm is left within the circulation.

We can apply the concept of the ideal closure line to aneurysms at any location, as the pathological basis for the origin and growth are supposed to be the same for all aneurysms. However, aneurysms at deeper locations than MCA aneurysms will be associated with more problems, such as limitations in approach angle. We are now investigating how to apply the concept of closure line to aneurysms at other locations.

\section{References}

1) Amagasaki $K$, Higa $T$, Takeuchi $N$, Kakizawa $T$, Shimizu T: Late recurrence of subarachnoid hemorrhage due to regrowth of aneurysm after neck clipping surgery. Neurol Med Chir (Tokyo) 42: 496-500, 2002

2) Dacey RG: Intracranial aneurysms: Surgical principles, in Batjer HH, Caplan LR, Friberg L, Greenlee RG, Kopitnik TA, Young WL (eds): Cerebrovascular Disease. Baltimore, New York, Lippincott-Raven, 1997, pp 901-914

3) Dashti R, Hernesniemi J, Niemela M, Rinne J, Porras M, Lehecka $M$, Shen $H$, Albayrak BS, Lehto $H$, Koroknay-Pal P, de Oliveila RS, Perra G, Ronkainen A, Koivisto T, Jaaskelainen JE: Microneurosurgical management of middle cerebral artery bifurcation aneurysms. Surg Neurol 67: 441-456, 2007

4) David CA, Vishteh AG, Spetzler RF, Lemole M, Lawton MT, Partovi S: Late angiographic follow-up review of surgically treated aneurysms. J Neurosurg 91: 396-401, 1999

5) Ishikawa $\mathrm{T}$ : [Clipping techniques for cerebral aneurysms. Special consideration for closure line 
and application angle]. No Shinkei Geka Sokuho 17: 804-814, 2007 (Jpn)

6) Kamitani H, Masuzawa H, Kanazawa I, Kubo T: Bleeding risk in unruptured and residual cerebral aneurysms - angiographic annual growth rate in nineteen patients. Acta Neurochir (Wien) 141: 153-159, 1999

7) Kang HS, Han MH, Kwon BJ, Jung SI, Oh CW, Han $\mathrm{DH}$, Chang $\mathrm{KH}$ : Postoperative 3D angiography in intracranial aneurysms. AJNR Am J Neuroradiol 25: 1463-1469, 2004

8) Kashimura H, Ogasawara K, Kubo Y, Ogawa A: Complete neck clipping of internal carotid-posterior communicating artery aneurysms using bayonet-shaped aneurysm clips. Technical note. Neurol Med Chir (Tokyo) 47: 282-284, 2007

9) Lin T, Fox AJ, Drake CG: Regrowth of aneurysm sacs from residual neck following aneurysm clipping. $J$ Neurosurg 70: 556-560, 1989

10) Nussbaum ES, Erickson DL: The fate of intracranial microaneurysms treated with bipolar electrocoagulation and parent vessel reinforcement. Neurosurgery 45: 1172-1175, 1999

11) Osawa M, Obinata C, Kobayashi S, Tanaka Y: Newly designed bayonet clips for complicated aneurysm: Technical note. Neurosurgery 36: 425-427, 1995

12) Rabinstein AA, Nichols DA: Endovascular coil embolization of cerebral aneurysm remnants after incomplete surgical obliteration. Stroke 33: 1809-1815, 2002

13) Sindou M, Acevedo JC, Turjman F: Aneurysmal remnants after microsurgical clipping: classification and results from a prospective angiographic study (in a consecutive series of 305 operated intracranial aneurysms). Acta Neurochir (Wien) 140: 1153-1159, 1998

14) Sundt TM Jr: 6 Basic principles and techniques, in: Surgical Techniques for Saccular and Giant Intracranial Aneurysms. Baltimore, Williams \& Wilkins, 1990, pp 39-56

15) Thornton J, Bashir Q, Aletich VA, Debrun GM, Ausman JI, Charbel FT: What percentage of surgically clipped intracranial aneurysms have residual necks? Neurosurgery 46: 1294-1300, 2000

16) Tsutsumi K, Ueki K, Usui M, Kwak S, Kirino T: Risk of subarachnoid hemorrhage after surgical treatment of unruptured cerebral aneurysms. Stroke 30: 1181-1184, 1999

17) Wermer MJ, Rinkel GJ, Greebe P, Albrecht KW, Dirven CM, Tulleken CA: Late recurrence of subarachnoid hemorrhage after treatment for ruptured aneurysms: patient characteristics and outcomes. Neurosurgery 56: 197-204, 2005

18) Yasargil MG: Aneurysm clipping. 3 General operative techniques, in Yasargil MG: Microneurosurgery I. Stuttgart, New York, Georg Thieme Verlag, 1989, pp 245-264
Address reprint requests to: Tatsuya Ishikawa, M.D., Department of Surgical Neurology, Research Institute for Brain and Blood Vessels-Akita, 6-10 Senshu-Kubota-machi, Akita 010-0874, Japan.

e-mail: teddyish@akita-noken.go.jp

\section{Commentary}

The authors present a very interesting topic on the concept of the ideal closure line for clipping of the middle cerebral artery (MCA) aneurysms. It is a well known rule that the ideal positioning of a clip over the "neck" of an aneurysm is such that the leg of the clip is aligned along the longitudinal axis of the parent artery. This is possible in most cases as long as the aneurysm is not located at a bifurcation or trifurcation of a parent artery. One of the most difficult location(s) where the positioning of the clip(s) is far from being easy is that of bifurcation and/or trifurcation of the MCA. Not only the geography of arterial branching but also the aneurysm location and changes in the wall(s) of the aneurysm and the branches of the MCA are of great importance. The surgeon is always perplexed with the requirements of the circulation through the MCA and its branches at the site of the aneurysm as well as beyond. Positioning of the clip(s) so that all weak points of the wall(s) are secluded on one side and confronting the sufficient (more or less normal) flow through the newly created vessel(s). What is less dangerous: too much clipping (reduced blood flow) or too little clipping (high probability of regrowth of the aneurysm and [new] SAH)? Shape of the clip(s), the length of the clip's leg, the angulation of the leg, its direction as well as many other characteristics of the clip(s) are also important. And what is also of important practical meaning is the fact that sometimes - or many times - on the "table" there are not enough varieties of the clips. Positioning of the "wrong" clip may result in insufficient blood flow or insufficient securing of the weak points of the wall(s). And that's why it is so important to study in advance the geography of the aneurysm and to be prepared as much as possible to solve the problem(s) accordingly. In my personal opinion, there are still cases where we cannot be $100 \%$ sure that everything is as it was thought in advance. And that's why the surgeon should always be prepared to abandon the idea of clipping and to perform reconstruction of the artery after resection of the aneurysm, either directly using the remnants of the wall(s) or by patching the opening of the defect at the bifurcation and/or trifurcation of the MCA. There might be neurosurgeons who will not agree with this; but what would be their answer when the arteriosclerotic plaque is found in the aneurysm 
which is the origin of the emboli? In many such cases no safe clipping is possible, and the excision of the diseased part of the atherosclerotic wall together with thrombus is necessary and again reconstruction of the MCA. The calcifications of the wall close to the aneurysm additionally complicate the whole situation. Needless to say, giant aneurysms do require special reconstruction techniques from which suturing reconstruction of the vessel(s) cannot be excluded. One only needs enough expertise with MCA aneurysms, then he/she cannot disagree that any - if not most - MCA aneurysms are not amenable for endovascular treatment. According to my personal opinion, the microsurgical approaches to MCA aneurysm are also not a task to be put on the residents' shoulders alone. Resection and suturing of small vessels in the depth is necessary for everybody who would like to tackle MCA aneurysms microsurgically. Courage only is not enough for both the surgeon and the patient. Unfortunately, too many times it satisfies only the surgeon.

Vinko V. DOLENC, M.D. Department of Neurosurgery University Medical Center Ljubljana, Slovenia

The authors have proposed the concept of 'ideal closure line' for clipping of the middle cerebral artery aneurysms in this technical note. This concept can be theoretically comprehended and accepted by most neurosurgeons with enough experience of aneurysm treatment, since they were already doing aneurysmal clipping based on a similar concept.

Middle cerebral artery aneurysms often have a broad neck. Particularly, the broad neck is more often seen in unruptured middle cerebral artery aneurysms than in ruptured ones, and the chance of operating unruptured middle cerebral artery aneurysms has recently increased. Therefore, the concept maintained in this study should be kept in mind during operation.

It is essential to image the final figure of clipping before the first clip is applied. The first clip should be placed so that the second clip can be placed easily. For example, if the dog-ear residual neck caused after the first clipping is too small to apply the second clip, the first clip should be placed so that the size of the dogear neck is appropriate for the second clipping.

The problem is that clipping along the ideal closure line could be done in only half of the cases in this report. In the cases in which the ideal clipping along the ideal closure line is difficult, we should not adhere to the concept of ideal closure line in order to avoid injury of the surrounding structures, such as the brain and blood flow in the arteries.

Hiroki OHKUMA, M.D. Department of Neurosurgery

Hirosaki University Graduate School of Medicine Hirosaki, Aomori, Japan

In this article, T. Ishikawa et al. report their experience in 41 patients harboring 51 MCA aneurysms of less than $15 \mathrm{~mm}$ in diameter treated by clipping. They applied the concept of ideal closure line to the clipping and demonstrated a combination clip technique based on their concept of aneurysm type and ideal closure line.

The goal of intracranial aneurysm treatment is the complete, permanent, and safe obliteration of the aneurysm with preservation of the parent artery and its branches. Therefore, the aim of direct surgical clipping is to incorporate the whole pathological wall of the aneurysm within the clip blades without compromising the parent artery and the adjacent neurovascular structures. Clipping is performed according to the assumed line determined preoperatively or intraoperatively. The concept of ideal closure line mentioned in this article is not a new concept.

Most small aneurysms, especially with smaller necks, can be completely occluded by a single clip. As the authors mentioned, clip application perpendicular to the efferent artery often results in dog-ear residual neck. In such cases, bipolar coagulation and combination clip techniques can be considered. Intermittent bipolar coagulation of low current is useful in shrinking and shaping the aneurysm with acceptable risk and permits more precise clipping. Various combination clip techniques are also useful. If available, intraoperative angiography is helpful to accomplish complete clipping without ischemic complications. It is important that the most appropriate clipping techniques must be determined according to the size and geometric configuration of the aneurysm and the adjacent neurovascular structures.

Young-Je Son, M.D. and Dae Hee HAN, M.D. Department of Neurosurgery

Seoul National University Boramae Medical Center Seoul, R.O.K. 